\title{
USE OF CROSS-POL MULTI-TEMPORAL SAR DATA FOR IMAGE SEGMENTATION
}

\author{
Claudio M. Prati, Fabio Rocca, Francesco Asaro, Barbara Belletti, Simone Bizzi
}

\author{
Politecnico di Milano (Italy)
}

\begin{abstract}
The contribution to the image segmentation of multitemporal SAR data acquired with VV - VH polarization is analyzed. The backscattering intensity, the short time interferometric coherence and the decorrelation time of scatterers have been used to separate different ground backscattering behavior both in $\mathrm{VV}$ and $\mathrm{VH}$ polarization. Then, these six parameters have been projected into a domain with reduced dimensionality by means of a Covariance Matrix Analysis. The resulting covariance values show that the main contribution to the image segmentation from the $\mathrm{VH}$ polarization comes when the short time interferometric coherence is different from that of the $\mathrm{VV}$ polarization. Preliminary results are presented on an agricultural area. Moreover, the VV-VH complex coherence has been exploited to identify urban areas and permanent scatterers. It has been verified that the coherence phase in urban environments can be only 0 or $\pi$ accordingly to the theory of dihedral and trihedral backscattering.
\end{abstract}

Index Terms- SAR, Land-use, Segmentation, Interferometry, Coherence

\section{INTRODUCTION}

In remote sensing applied to land use monitoring, Synthetic Aperture Radar (SAR) is able to provide medium/high resolution, all-weather, day and night, coherent data which is complementary to optical data. The availability of Sentinel 1 A and B SAR satellites data of the European Copernicus program represents an important new tool for Earth Observation. The decision to select TOPS as the standard background acquisition mode for both Sentinel $1 \mathrm{~A}$ and B of the European Copernicus program is strategic for Earth observation. It offers the unique possibility to get SAR images of the Earth surface every 6 days almost everywhere with a spatial resolution of about 5 by 20 meters in EastWest and North-South directions. Thanks to the regular availability of Sentinel data, new scattering features of the Earth surface can be identified by analyzing long time series of SAR images with given incidence. Averaged time series SAR data (intensity and coherence) are usually combined to support land use classification purposes (e.g. Armitrano et al. 2016). In the present paper, the intensity of backscattering, the short time interferometric coherence and the decorrelation time of ground scatterers [1] are exploited to identify different backscattering features possibly connected to specific ground characteristics.

\section{METHODOLOGY}

\section{A. Backscattered intensity}

The backscattered intensity is very high for solid scatterers that generate dihedral/trihedral reflection toward the radar as in the case of urban environments. It is low for calm water due to specular reflection. It has intermediate decreasing values for vegetation, agricultural fields and bare soil. In all cases the backscattered intensity is affected by the so called "speckle noise" (that actually is not at all noise, but useful signal for interferometry). The speckle has the effect to spread the intensity histogram of each class so mixing one class with the other.

Speckle reduction can be achieved with no spatial resolution loss by averaging as many detected SAR images as possible taken on similar weather and on almost unchanged ground conditions (the image stack range of dates is then limited to at least the same season). Moreover, homogeneous pixels can be identified from the data stack and spatially averaged so further reducing the speckle effect. The averaged amplitude computed on an 18 image stack of $\mathrm{VV}$ and $\mathrm{VH}$ polarized Sentinel-1 images over an agricultural area of about 3 by $4 \mathrm{~km}$ in the Padania plains is shown in the upper part of figure 1. Due to the very large dynamic of the SAR image intensity, values are saturated to 3 times the deviation and rescaled in the range 0 to 1 . All the images have been acquired during the very dry summer period 2017 from June $4^{\text {th }}$ to September $14^{\text {th }}$ and apart from some worked fields the ground condition they can be considered in an almost unchanged status. In the lower part of figure 1 the optical image of the same area taken from Google maps is shown for the sake of comparison. Borders of agricultural fields can be easily identified both on optical and despeckled images.

\section{B. Coherence and decorrelation time}

Coherence is defined as the local complex correlation coefficient of two SAR images $I_{1}$ and $I_{2}$ after removal of the deterministic interferometric phase contribution $\varphi$ due 
to the normal baseline, possible orbit crossing effects and topography. The complex coherence $\gamma$ is computed as in eq. 1 convolving numerator and denominator with a small Gaussian elliptical window $W(n, m)$ that achieves a smooth moving average effect on almost 80 independent pixels.

$\gamma(n, m)=\frac{W(n, m) *\left(I_{1} I_{2}^{*} e^{-j \varphi}\right)}{W(n, m) *\left|I_{1}\right|\left|I_{2}\right|}$

In eq. 1 the symbol $*$ stays for convolution.

The 18 image stack is used to compute the complex coherence for 6 different time intervals at $6,12,18,24,30$ and 36 days. Then the simple negative exponential temporal decorrelation model of eq.2, as suggested in [1], is assumed for all the scatterers of the image.

$$
\gamma(t)=\gamma_{0} e^{\frac{-t}{\tau}}
$$

A very long decorrelation time $\tau$ is expected for urban environments and solid "permanent scatterers" [2]. Shorter and shorter decorrelation time is expected passing from bare soil to agricultural field to vegetation and water. As far as the short term coherence $\gamma_{0}$ is concerned, it is expected again very high for urban environments and "permanent scatterers", moderate for agricultural field and low for dense vegetation and water. The short term coherence $\gamma_{0}$ and the decorrelation time $\tau$ can be estimated for each pixel of the image, by linear fitting the computed 6 coherence values in a logarithmic scale as shown in eq. 3 .

$$
\log (\gamma(t))=\log \left(\gamma_{0}\right)-\frac{t}{\tau}
$$

\section{AMPLITUDE, COHERENCE AND DECORRELATION TIME COMBINATION}

De-speckled intensity, short term coherence $\gamma_{0}$ and the inverse of the decorrelation time $\tau$ (its value ranges between 0 for permanent scatterers and large values for fast decorrelating scatterers) are then scaled so that their dynamic range is limited between 0 and 1 . These values are then used to form the composite RGB images shown in figures 2 and 3 for the $\mathrm{VV}$ and the $\mathrm{VH}$ polarizations respectively. The RGB images of figures 2 and 3 show that the combination of these six components (three for $\mathrm{VV}$ and three for $\mathrm{VH}$ polarization) could be exploited to form a robust indicator of the different land use in the imaged area. A quantitative measure of the information added by each of these six components with respect to the others is given by their Covariance matrix.
As an example the covariance matrix of the agricultural area of figure 1 is shown in the following Table 1 .

From the covariance matrix of Table 1, a high covariance value $(0,81)$ between the short term coherence components carried out in VV and VH polarization is observed. Such a high covariance value means that the added information of the cross-pol component is not relevant at least in the average of the observed scene. However, the cross-plot between $\gamma_{0} \mathrm{VV}$ and $\gamma_{0} \mathrm{VH}$ of figure 4 shows two clearly distinct families of scatterers with a different behavior. The location of the two classes of scatterers that belong to the elliptical areas of figure 4, are shown in figure 5. Those scatterers that belong to the lower left ellipse of figure 4 are shown in gray, whereas those belonging to the upper right ellipse are shown in white. Black areas represent pixels that fall outside both ellipses.
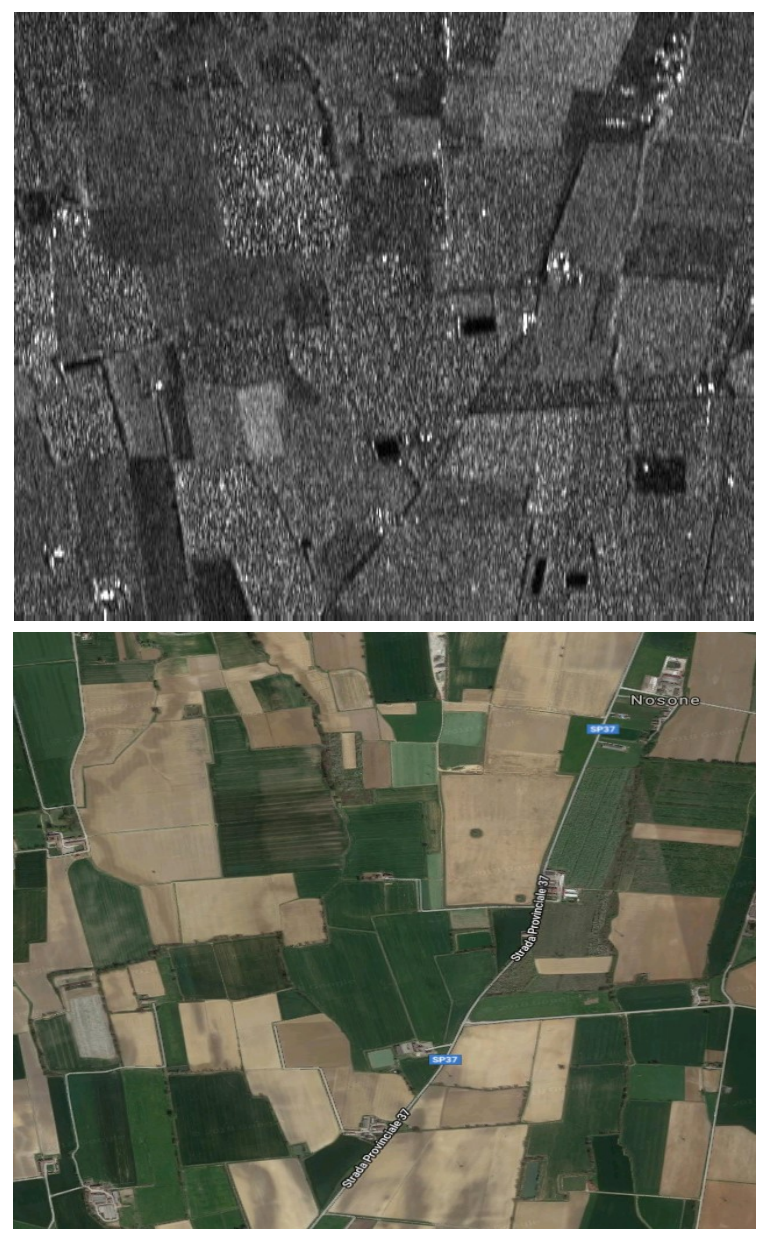

Figure -1 Upper image: average amplitude computed on an 18 image stack over a 3 by $4 \mathrm{~km}$ agricultural area in the Padania plains. Lower image: Google maps optical image of the same area. 
The shape of gray and white areas of figure 5 resemble rather precisely the shape of the agricultural fields shown in figure 1. Thus, the local behavior of the cross-pol coherence could be possibly used as an addition tool for image segmentation and land use classification.

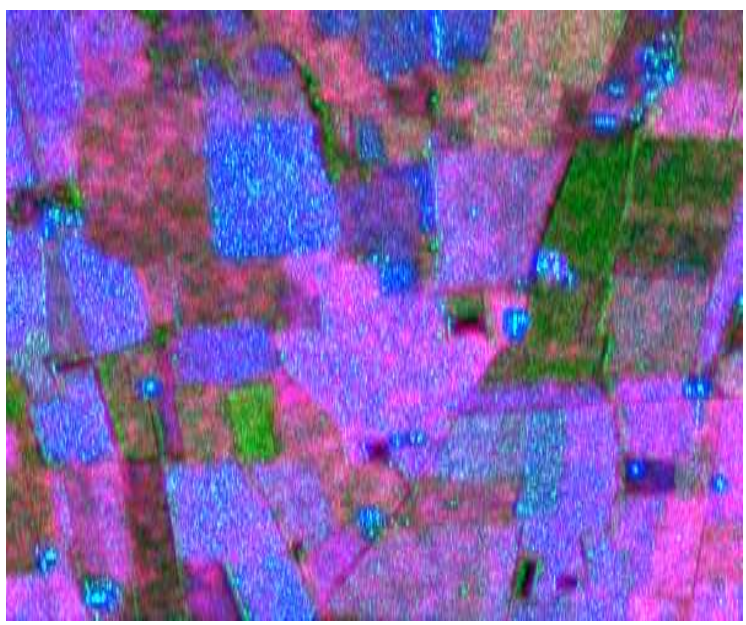

Figure -2 False color RGB image formed by the rescaled values of the average intensity, short term coherence $\gamma_{0}$ and inverse decorrelation time $1 / \tau$ computed from the $\mathrm{VV}$ polarized stack.

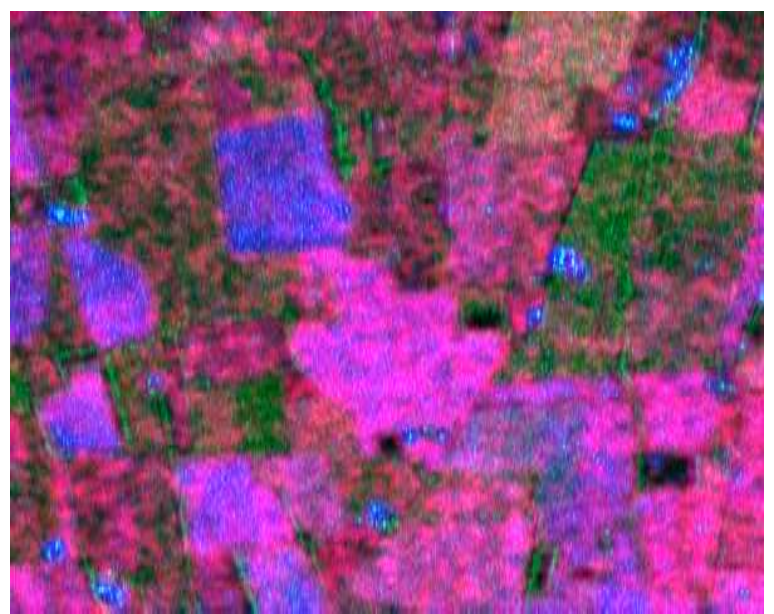

Figure -3 False color RGB image formed by the rescaled values of the average intensity, short term coherence $\gamma_{0}$ and inverse decorrelation time $1 / \tau$ computed from the $\mathrm{VH}$ polarized stack.

\begin{tabular}{|l||c|c|c|c|c|c|}
\hline & $\mathrm{I}-\mathrm{VV}$ & $\gamma_{0} \mathrm{VV}$ & $1 / \tau \mathrm{VV}$ & $\mathrm{I}-\mathrm{VH}$ & $\gamma_{0} \mathrm{VH}$ & $1 / \tau \mathrm{VH}$ \\
\hline \hline $\mathrm{I}-\mathrm{VV}$ & $\mathbf{1}$ & 0.15 & -0.04 & 0.32 & 0.21 & 0.07 \\
\hline \multicolumn{1}{l|}{$\gamma_{0} \mathrm{VV}$} & 0.15 & $\mathbf{1}$ & 0.33 & -0.28 & 0.81 & 0.52 \\
\hline $1 / \tau \mathrm{VV}$ & -0.04 & 0.33 & $\mathbf{1}$ & -0.15 & 0.17 & 0.54 \\
\hline $\mathrm{I}-\mathrm{VH}$ & 0.32 & -0.28 & -0.15 & $\mathbf{1}$ & -0.03 & -0.14 \\
\hline \multicolumn{1}{l}{$\gamma_{0} \mathrm{VH}$} & 0.21 & 0.81 & 0.17 & -0.03 & $\mathbf{1}$ & 0.6 \\
\hline $1 / \tau \mathrm{VH}$ & 0.07 & 0.52 & 0.54 & -0.14 & 0.6 & $\mathbf{1}$ \\
\hline
\end{tabular}

Table 1 - Covariance matrix of the agricultural area shown in figure 1 .

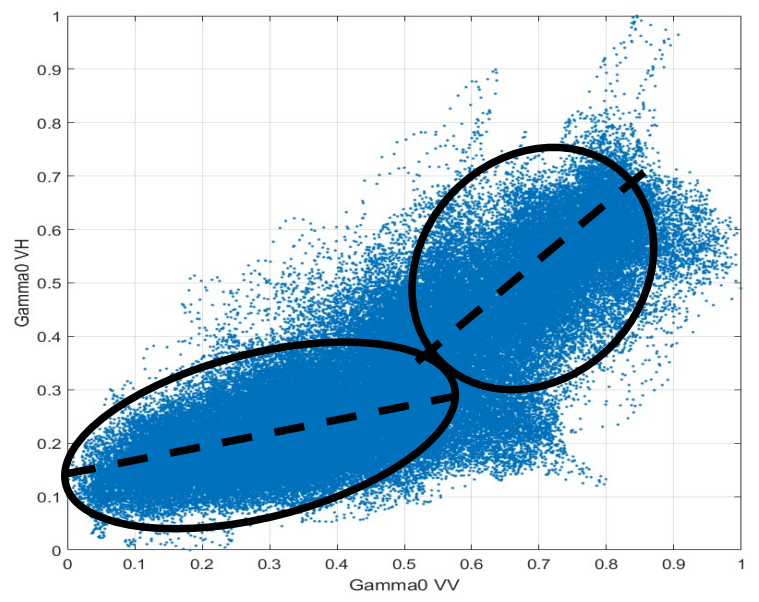

Figure - 4 Cross-plot between $\gamma_{0} \mathrm{VV}$ and $\gamma_{0} \mathrm{VH}$. Two distinct families of scatterers are identified with two elliptical areas.

\section{USE OF COMPLEX VV/VH COHERENCE IN URBAN ENVIRONMENTS}

The complex cross-pol coherence can be defined as the local cross-correlation between simultaneously acquired VV and VH SAR images. It can be computed in practice as shown in eq. 4 :

$\gamma_{V V-V H}(n, m)=\frac{W(n, m) *\left(I_{V V} I_{V H}^{*}\right)}{W(n, m) *\left|I_{V V}\right|\left|I_{V H}\right|}$ 
where $W(n, m)$ is a gaussian elliptical window that achieves a smooth moving average effect on almost 10 independent pixels. The symbol $*$ stays for convolution. In this case a small Gaussian window has be adopted since the cross-pol coherence is used to detect buildings rather than agricultural fields.

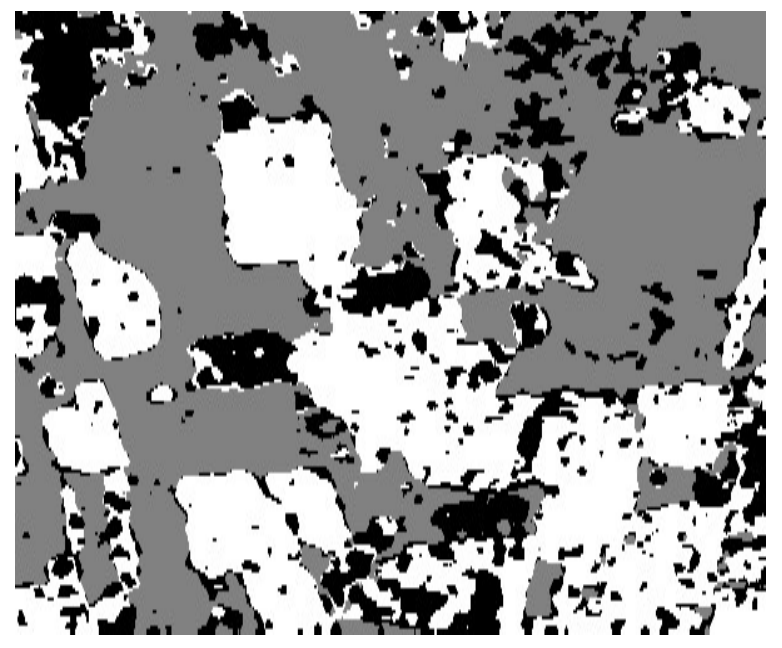

Figure 5 - Space location of scatterers that belong to the lower left ellipse of figure 4 (gray), and to the upper right ellipse of figure 4 (white). Black areas represent pixels that fall outside both ellipses.

In [3] it has been demonstrated that the strong radar backscatterer observed in urban environments is generated by dihedral double bouncing. It is the nonplanar aspect of the ground and the walls of buildings that leads to double bounce reflections. In [3] it has also been demonstrated that the cross-pol phase (XPD) of these oriented dihedrals can assume only 2 values: 0 or $\pi$. In other words, the complex coherence computed between the co and cross-pol components by means of eq. 4 shows only real positive or negative values close to 1 and -1 in correspondence of buildings. Moreover, the sign of this real coherence values can be used to discern clockwise $(\mathrm{CW})$ or counterclockwise (CCW) horizontal orientation of the dihedrals with respect to the SAR line of sight (LOS). These properties of the cross-pol coherence can be thus exploited to improve the classification of urban areas from SAR images. The theory is confirmed by the experimental results achieved by using sentinel 1 SAR images acquired simultaneously in VV and $\mathrm{VH}$ polarization. The selected area used for testing is the city of Milano and its neighboring smaller villages. SAR and optical Google images of the selected area are shown in figure 6. The complex cross-pol coherence $\gamma_{V V-V H}(n, m)$ has been computed by applying eq. 4 to a single pair of $\mathrm{VV}$ and VH Sentinel-1 images. Its real value is shown in figure 7. The very high statistical background noise caused by the reduced Gaussian window size used in eq.4 is clearly visible and could be a major problem for automatic image segmentation. However, the power of this background statistical noise can be reduced by a factor of $\mathrm{N}$ by averaging $\mathrm{N}$ independent realizations of the cross-pol coherence in which buildings remain unchanged. The effect of such a background noise reduction is shown in figure 8 where 18 realizations of the cross-pol coherence covering four-month time period have been averaged. In figure 8 it can be noted that \pm 1 cross-pol coherence values appear in correspondence of buildings and other man-made dihedral structures.
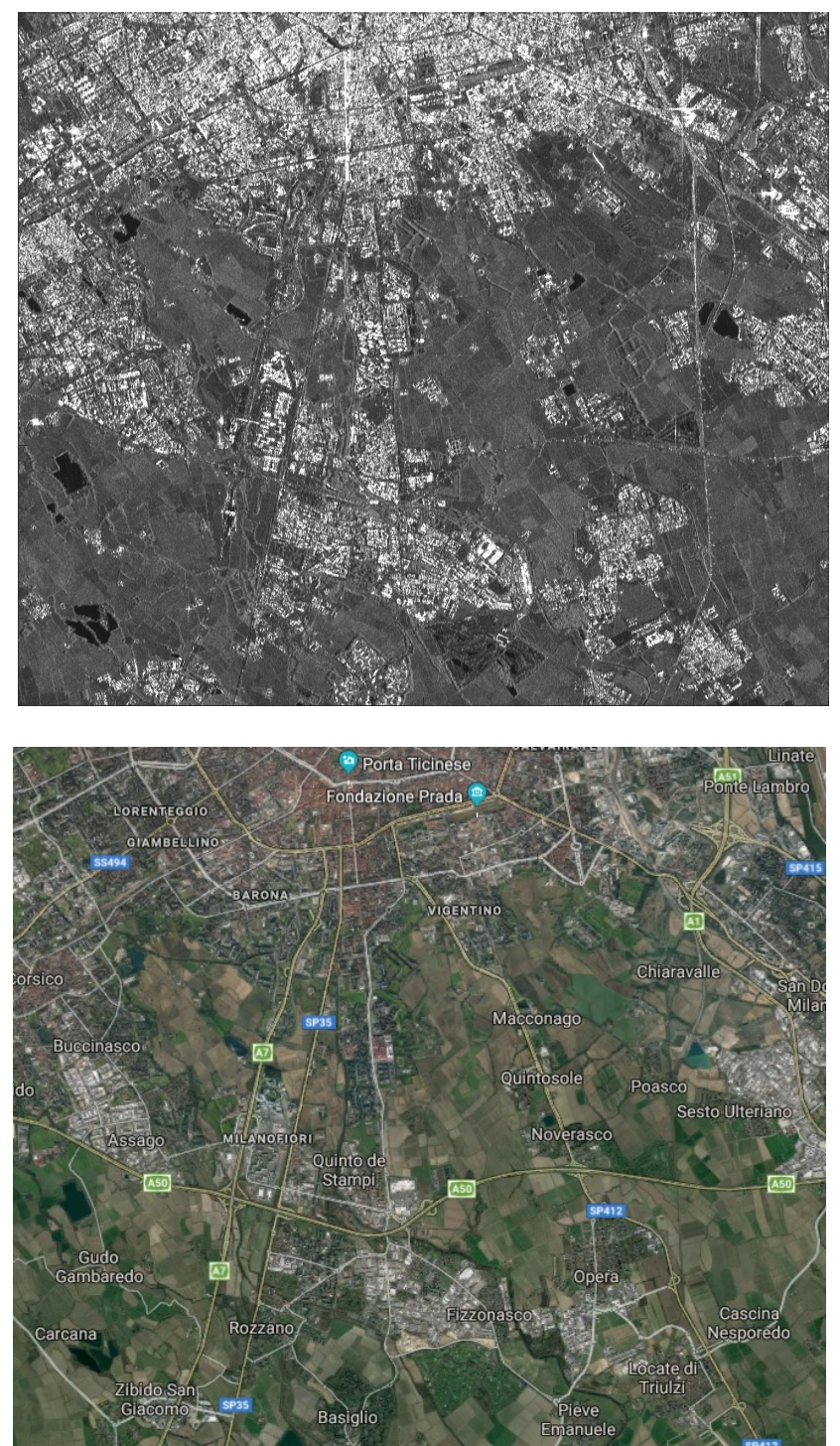

Figure 6 - Upper image: average amplitude computed on an 18 image stack over the southern part of Milano and neighboring smaller villages. Lower image: Google maps optical image of the same area. 


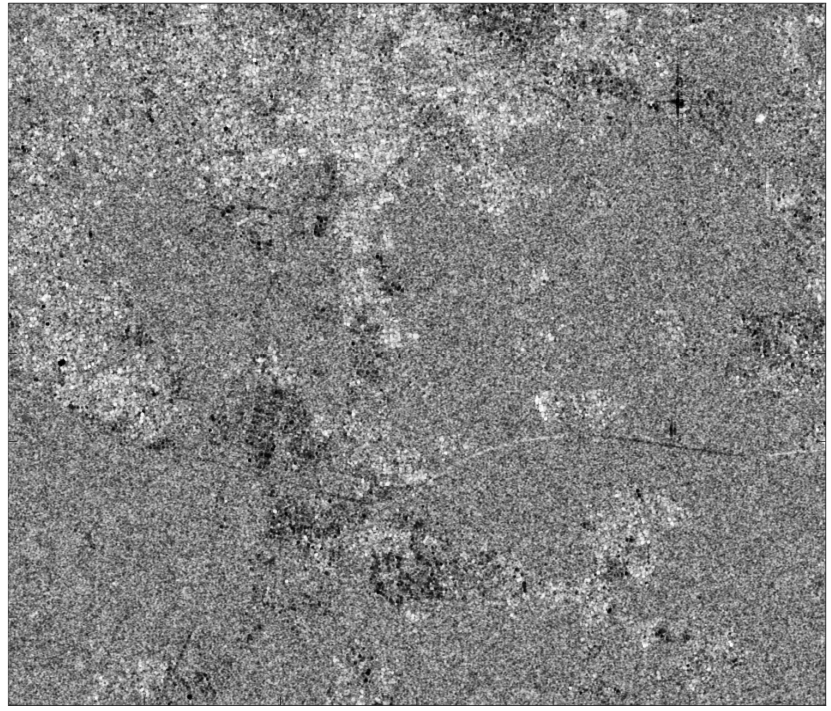

Figure 7 - Real value of the cross-pol coherence of the area of Milano shown in figure 6 computed on a single $\mathrm{VV} / \mathrm{VH}$ Sentinel-1 image pair. The gray scale range from -1 (black) to +1 (white).

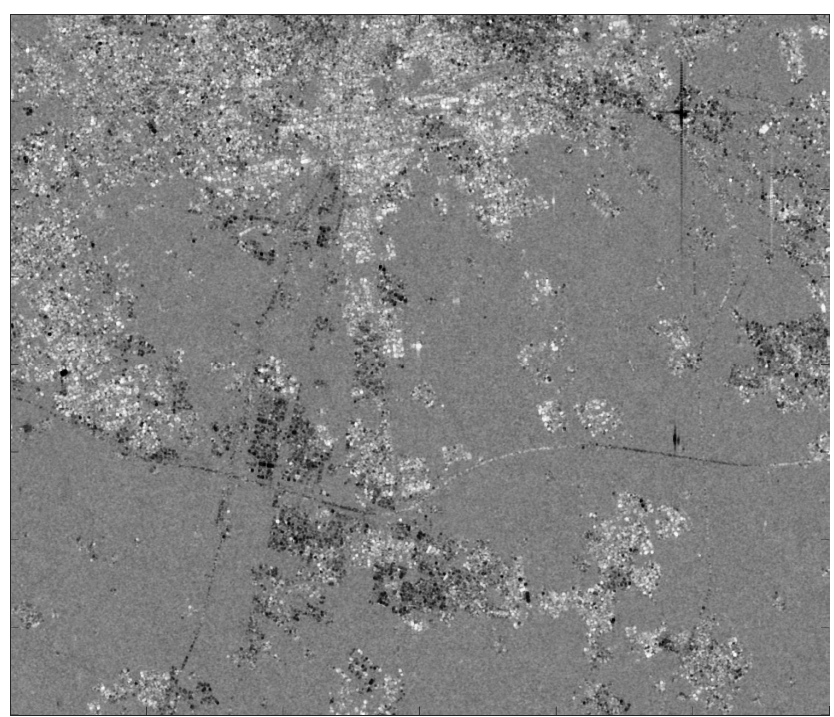

Figure 8 - Real value of the 18 cross-pol coherence average. The same gray scale of figure 7 ranging -1 (black) to +1 (white) has been adopted.

In particular, it is quite interesting to observe the cross-pol values transition from -1 (black) to +1 (white) following the curvilinear "A50" highway track that crosses horizontally the image. The dihedrals structures formed by guardrails and the asphalt change their orientations with respect to the SAR line of sight moving from the left $(\mathrm{CW})$ to the center $(\mathrm{CCW})$ and to the right $(\mathrm{CW})$ of the image. The same $\mathrm{CW} / \mathrm{CCW}$ orientation effect can be observed on the dihedrals formed by the ground and the walls of buildings in urban areas. Figure 9 shows the optical Google image of the center of Milano and the correspondent real part of the cross-pol coherence.
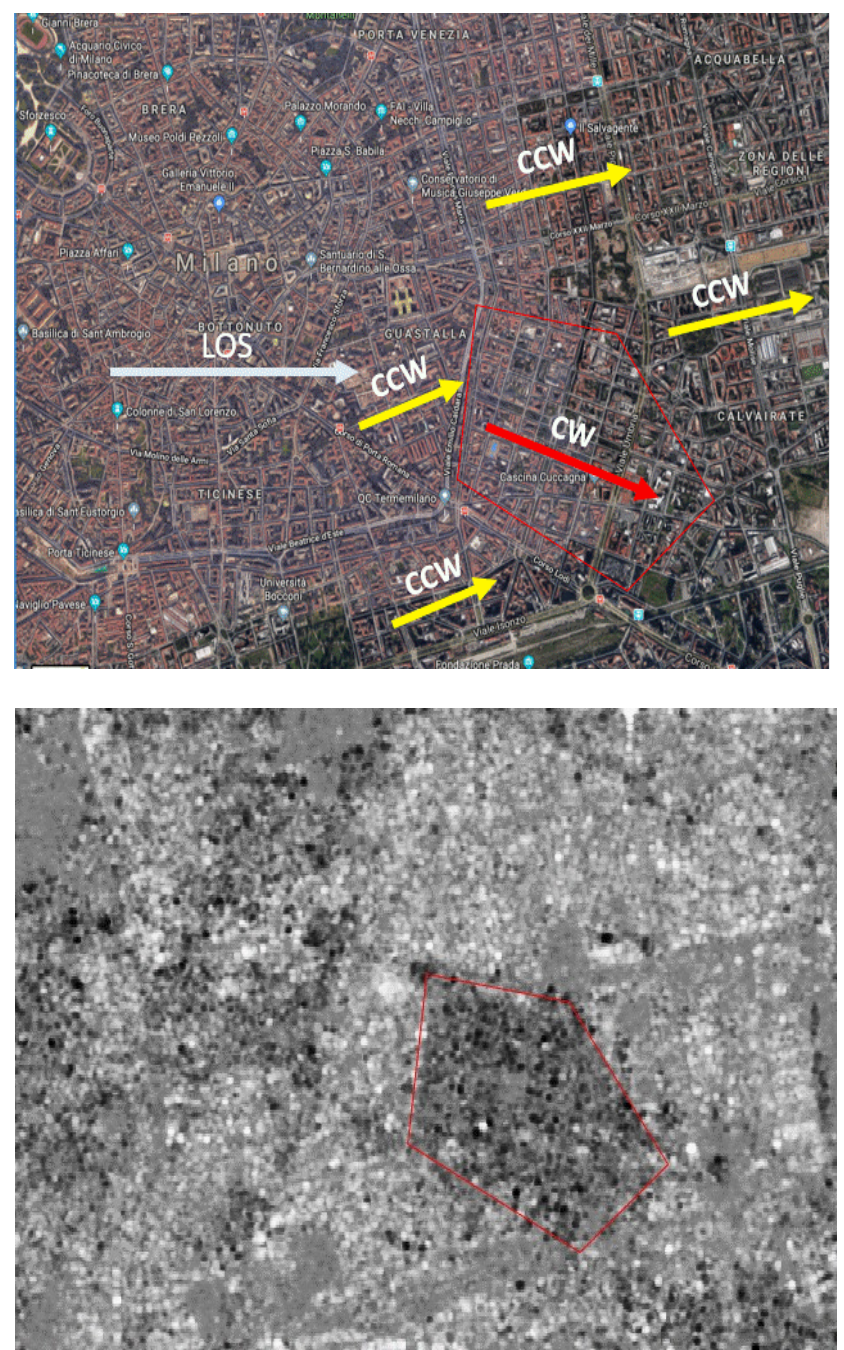

Figure 9 - Upper image: optical Google image of the center of Milano. Lower image: the correspondent real part of the cross-pol coherence.

The CW and CCW orientation of the walls of buildings with respect of the SAR line of sight (the LOS arrow in figure 9) produces -1 (black) and +1 (white) value of the cross-pol coherence. From the optical image of figure 9 it can be noted that the buildings within the shown polygon are clearly $\mathrm{CW}$ oriented since their reflecting walls are parallel to the streets. Instead the buildings surrounding the polygon are CCW oriented. average. The very same buildings orientation scheme can be observed by the cross-pol image as predicted by the theory. 


\section{CONCLUSIONS}

An interesting and promising approach for image segmentation and possibly for land use classification comes from the combination of different data derived from multitemporal VV and VH SAR data. These type of data are regularly provided by the Copernicus Sentinel missions every 6 days on a large portion of the Earth. In particular, it has been shown that de-speckled intensity, short time VV and $\mathrm{VH}$ coherence and decorrelation time together with the time-averaged cross-pol coherence can be exploited to separate different types of land cover and land use.

Future classification techniques should explore how these different variables change for different temporal image stacks chosen in function of seasonal, climate and anthropic factors (e.g., management measures). This step could open new opportunities towards more process based classifications [4] of lands and landscapes.

The combination of these data with optical information from, for instance, Sentinel-2, the multi-spectral optical satellite of the Copernicus program, will improve the quality and frequency of classifications [5]. For instance, the allweather and day and night availability of SAR data could integrate the land use monitoring of artificial areas that are commonly based on optical-derived metrics (e.g. imperviousness index [6]). A more detailed analysis of the segmentation capability offered by these data should be carried out by an extensive principal components analysis covering different areas and different seasons.

\section{REFERENCES}

[1] Rocca, F. "Modeling Interferogram Stacks" " IEEE Transactions on Geoscience and Remote Sensing, , Volume:. 45, no. 10, pp. 3289-3299, October 2007.

[2] Ferretti A., Prati, C., Rocca, F., "Permanent Scatterers in SAR Interferometry" IEEE Transactions on Geoscience and Remote Sensing, Volume: 39, Issue: 1, pp. 8-20, Jan 2001.

[3] Atwood D. K., Thirion-Lefevre, Laetitia, "Polarimetric Phase and Implications for Urban Classification", IEEE Transactions on Geoscience and Remote Sensing, pp. 1-12, 10.1109/TGRS.2017.2750211.

[4] Cullum, C., K. H. Rogers, G. Brierley, e E. T. F. Witkowski. "Ecological classification and mapping for landscape management and science: Foundations for the description of patterns and processes", Progress in Physical Geography, 2015. https://doi.org/10.1177/0309133315611573.

[5] Ullmann, T.; Schmitt, A.; Roth, A.; Duffe, J.; Dech, S.; Hubberten, H.-W.; Baumhauer, R., "Land cover characterization and classification of arctic tundra environments by means of polarized synthetic aperture X- and C-Band Radar (PolSAR) and Landsat 8 multispectral imagery-Richards Island, Canada", Remote Sens. 2014, 6, 8565-8593

[6] https://land.copernicus.eu/pan-european/high-resolution-layers/ imperviousness/imperviousness-2012/view 